评 述纳米结构生长机理专刊

\title{
Janus 材料微结构精细调控进展
}

\author{
张成亮，韦玮，梁福金金，杨振忠 ${ }^{*}$ \\ 高分子物理与化学国家重点实验室; 中国科学院化学研究所, 北京 100190 \\ *通讯作者, E-mail: yangzz@iccas.ac.cn
}

收稿日期: 2012-01-07; 接受日期: 2012-04-18; 网络版发表日期: 2012-07-11 doi: 10.1360/032012-3

\begin{abstract}
摘要 Janus 材料由于其拥有两个具有不同化学组成的表面而备受关注，其特殊的结构和 性能已成为材料科学研究热点. 如何实现Janus 材料形貌可控、化学组成严格分区、不同位 点复合功能分区、微结构精细调控和批量化制备是该研究方向中的重点和难点. 针对上诉 问题, 基于本课题组研究工作, 本文总结了 Janus 材料微结构精确控制和批量化制备的方 法, 为 Janus 材料大规模制备和应用提供新思路和新方法.
\end{abstract}

关键词

Janus 材料

各向异性

微结构精细调控

批量制备

\section{Janus 材料的典型性质与应用}

古罗马神 Janus 的头部具有双面结构, 分别朝向 过去和未来. P. G. de Gennes 于 1991 年在诺贝尔颁奖 演讲中, 首次借用 Janus 一词描述同一颗粒两面具有 不同的化学组成或性质, 并预测 Janus 颗粒类似双亲 性分子可在液/液界面自组装, 颗粒间的缝隙为物质 在两相间传输提供了通道. de Gennes 充满启发性的 演说引发了 Janus 颗粒的研究热潮 ${ }^{[1 \sim 6]}$. Janus 材料种 类日益丰富, 其特殊性质和诱人应用前景不断展现, 涉及物理、化学、材料、生物等多个领域.

\section{1 颗粒乳化剂}

1907 年, Pickering ${ }^{[7]}$ 发现固体颗粒具有稳定油/水 乳液的作用, 称之为 Pickering 乳液. 与传统小分子表 面活性剂和聚合物乳化剂的乳化机理不同, 虽然固 体颗粒并不能降低界面张力 ${ }^{[8]}$, 但其在油/水界面具 有高吸附能, 可保证 Pickering 乳液稳定, 这种稳定性 与颗粒的化学性质(表面张力)和尺寸有关 ${ }^{[9]}$. 传统表 面活性剂则是利用其双亲特性降低油/水界面张力实 现乳液稳定. 如果能够使固体颗粒的一部分表面亲 水, 而另一部分表面同时亲油, 制备 Janus 粒子, 就
可以将固体颗粒的 Pickering 效应与分子表面活性剂 降低界面张力的能力结合起来, 强化其乳化能力和 乳液稳定性. Binks 等 ${ }^{[10]}$ 发现, Janus 粒子的脱附能是 普通粒子的 3 倍, 且当平均接触角为 $0^{\circ}$ 或 $180^{\circ}$ 时, Janus 粒子仍能保持很强的吸附力, 表明 Janus 粒子能 够更好地稳定乳液. 与普通颗粒相比, Janus 颗粒在油 水界面能更好地降低界面张力, 乳化作用更强 ${ }^{[11]}$. Krausch 等 ${ }^{[12]}$ 通过研究 Janus 粒子在液-液界面的吸附, 从实验上证实了 Binks 的上述研究. Müller 等 ${ }^{[13]}$ 用聚 苯乙烯/聚甲基丙烯酸甲酯(PS/PMMA)Janus 粒子代 替分子乳化剂, 在极低的 Janus 粒子浓度下实现了苯 乙烯单体的常规乳液聚合. 这种 Janus 粒子可用作 PS 和 PMMA 共混物界面相容剂(图 1) ${ }^{[14]}$, 即使在高温和 高剪切作用下, 颗粒仍能稳定地吸附在界面, 表现出 优异的增溶特性.

表面活性剂分子结构和亲水-亲油平衡(HLB)值 决定了其相互作用的热力学与动力学及其界面行为 和超分子组装结构. 类似地, 对于 Janus 粒子, 形状 控制和亲水-亲油平衡性的控制同样重要. 球形颗粒 具有高度对称性, 而 Janus 片状材料在化学组成与形 貌具有高度各向异性, 具有更加丰富的相行为. Janus 片状材料的吸附能要比表面活性剂分子的吸附能高几 


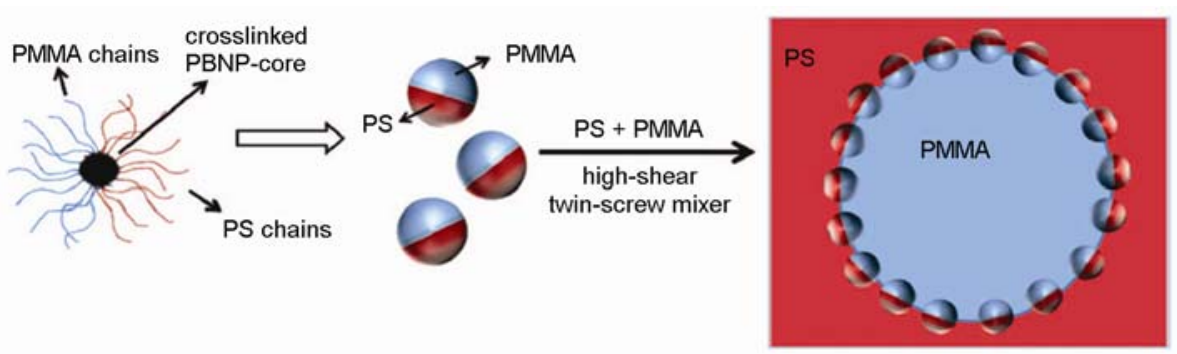

图 1 PS/PMMA Janus 颗粒作为相容剂增容 PS/PMMA 共混体系 ${ }^{[14]}$

个数量级; 与球形 Janus 颗粒相比, 各向异性结构的 Janus 片在乳液界面的旋转受到限制, 片状材料稳定的 乳液比其他结构颗粒稳定的乳液的稳定性高 $\left.{ }^{[15}, 16\right]$. 亲 水-亲油平衡(HLB)值是表面活性剂分子的重要参数, 对于颗粒也存在着称为 Janus 平衡性的参数(Janus Balance $)^{[17]}$, 体现了颗粒的亲水-亲油的相对强度, 与 不同化学组成分区的分区面积和化学组成有关, 可为 材料结构设计和应用选择提供参考. Granick 等 ${ }^{[18]}$ 通过 控制 Janus 颗粒表面不同化学分区面积实现了 Janus 平衡性参数的调节.

\section{2 自驱动马达}

Janus 颗粒可作为自驱动马达 ${ }^{[1925]}$. 当 Janus 颗 粒某区域的物质与体系中物质发生化学反应导致局 部微环境发生变化, 如生成气泡, 可产生能量推动 Janus 颗粒运动. 如通过 Janus 颗粒表面 Pt 催化过氧 化氢分解产生氧气, 实现颗粒的自驱动, 这种运动在 短时间内是有序的 ${ }^{[19]}$. 如果将 Janus 颗粒进行功能化, 如修饰具有靶向功能物质, 将为药物的高效输送提 供新思路.

\section{3 光学探针}

Janus 颗粒一侧半球表面为金属, 能够在不同方 向上反光, 随着表征聚合物体系的结构变化而变化, 反映了体系流变学性质. Behrend 和 Kopelman 等 ${ }^{[26 ~ 29] ~}$ 将 Janus 颗粒作为光学探针, 研究了受限空间微流变 行为和相互作用. Choi 等 ${ }^{[30]}$ 则用这种 Janus 粒子探针 研究了分子间相互作用. Sun 等 ${ }^{[31]}$ 对亚铃形 Janus $\mathrm{Fe}_{3} \mathrm{O}_{4} / \mathrm{Au}$ 的粒子进行改性, 用作光学和磁响应双模 生物探针.

\section{4 彩色显示}

将不同颜色引入到 Janus 粒子表面的不同微区,
并辅以 Janus 颗粒各向异性的磁或电响应，可用于彩 色显示. Nisisako 等 ${ }^{[32,33]}$ 制备了表面分别涂有黑白颜 料的 Janus 粒子, 置于电极间, 改变电场方向实现了 平板黑白显色. 将荷电粒子装入微胶囊中, 改变电场 控制粒子旋转, 可实现彩色显示 ${ }^{[33]}$. 同理, Janus 颗粒 可制备彩色电子纸 ${ }^{[34,35]}$. 具有苂光、磁响应性的 Janus 颗粒可用于大面积标识、手持柔性显示器等 ${ }^{[36]}$.

\section{5 自组装}

Janus 粒子是一种固体表面活性剂, 与表面活性 剂分子能组装不同的超分子聚集态结构类似, Janus 粒子也能组装成超结构, 其动态行为和结构可借助 显微镜直接观察. Granick 等 ${ }^{[3741]}$ 在该领域开展了大 量工作, 利用苂光显微镜直接观察到的 Janus 粒子组 装结构, 与计算机模拟结果一致 ${ }^{[38]}$. 一边带有负电荷, 另一边疏水的 Janus 微球在水中的组装结构由体系中 盐浓度控制, 在没有外加盐时, 其为单个颗粒; 在低 盐浓度时, 其为少于十个颗粒组成的聚集体; 在高盐 浓度时, 则形成了蠕虫状链的球簇 ${ }^{[39]}$. 三组分/微区 的 Janus 颗粒组装得到双亲超结构多孔材料, 部分孔 洞微区亲水, 部分孔洞微区亲油 ${ }^{[40]}$, 为制备选择性多 孔膜材料提供了新思路.

\section{Janus 材料可控制备}

早在 1985 年, Lee 等 ${ }^{[42]}$ 就通过种子乳液聚合制备 了具有双面性的 PS/PMMA 非球形乳胶颗粒. 后来, Casagrande 描述了二维平面保护法制备毫米级 Janus 玻璃球, 裸露部分以十八烷基三氯硅烷改性赋予其 亲油特性 ${ }^{[43,44]}$. Janus 玻璃球两面分别具有亲水和亲 油性质, 具有稳定乳液的作用, 玻璃球在油水界面排 列具有方向性.

近 20 年来, Janus 材料得到了高速发展, 展示了 
诸多新颖性质和诱人的应用前景, 尽管相关材料的 制备方法和性能研究方面取得了重要进展 ${ }^{[1 \sim 6]}$, 但现 存制备 Janus 材料的方法仍然存在问题. 例如: 最常 用的界面保护法虽能实现 Janus 材料结构精确控制, 但难以批量化生产; 微流体法制备的 Janus 材料组成 严格分区且形态多样, 但尺寸较大, 无法获得亚微米 甚至纳米尺度的材料; 模板法过程复杂, 制备效率低. 目前易于批量化生产的方法是相分离法和界面成核 法, 但制备的 Janus 材料难以实现化学组成的严格分 区和微结构的精细控制. 因此, 目前仍严重缺乏 Janus 颗粒组成、形貌及化学组成严格分区和微结构 的精细调控方法, 大批量制备 Janus 颗粒仍是制约其 广泛应用的最大瓶颈. 为此, 我们课题组从 2003 年 开始了相关的基础研究工作. 本综述围绕上述关心 的问题加以总结.

\subsection{Pickering 乳液界面制备 Janus 颗粒}

在制备 Janus 颗粒过程中经常需要对颗粒部分保 护, 以界面将颗粒分为两部分后, 可分别对其进行选 择性化学改性或者功能物质复合. 二维界面保护方 法简单直接, 产物组成和结构精确可控, 但产量极低. 界面保护方法进一步拓展到三维球面, 可显著提高 Janus 颗粒的制备产量. 对固体颗粒适当化学改性使 之具有合适的亲油或亲水性质, 则其能作为固体乳 化剂在油/水界面稳定形成 Pickering 乳液 ${ }^{[7]}$, 为颗粒 在界面处的选区保护和选择性化学修饰提供了条件, 为进一步高效制备 Janus 颗粒提供了可行性. Granick 等 ${ }^{[39,40,45]}$ 最早报道了 Pickering 乳液界面法制备 Janus 颗粒的研究. 以改性二氧化硅颗粒作为乳化剂, 在高 温下乳化石蜡/水体系, 形成水包油型 Pickering 乳液. 为了避免颗粒界面处转动导致的化学不对称改性失 败, 采取降温方法使石蜡凝固, 二氧化硅颗粒被镶嵌 在石蜡球表面. 再用硅烷偶联剂改性裸露在外的二 氧化硅表面, 除去石蜡后, 即可得到 Janus 颗粒 ${ }^{[45]}$. 外加表面活性剂调节颗粒在石蜡/界面处嵌入油相和 水相的深度 ${ }^{[39,40]}$, 可进一步调节 Janus 颗粒亲水/亲油 的相对比例. 虽然该方法实现了对颗粒表面的化学 组份进行功能分区, 但是缺乏形状控制, 得到的 Janus 颗粒通常为球形. 非球形 Janus 颗粒的相互作用 与几何空间的受限耦合作用, 在组装行为方面表现出 特殊性, 如中间带有磁性环的不对称哑铃状非球形 粒子在磁场下能组装出手性螺旋结构 ${ }^{[46]}$. 磁场与表
面化学共同提供了方向性的相互作用力, 而不对称 的空间位阻提供了链旋转的必要条件, 二者缺一不 可 ${ }^{[47]}$. 而球形的 Janus 胶体无论是在液相中, 还是在 外场中只能得到一些簇和链状结构 ${ }^{[48]}$.

我们提出了 Pickering 乳液界面颗粒化学刻蚀制 备非球形 Janus 颗粒的新方法(图 2(a)) ${ }^{[49]}$. 改性的二 氧化硅颗粒牢固地镶嵌在石蜡球表面, 对其裸露在 水相部分进行化学刻蚀, 去除表面一层功能物质重 新裸露出二氧化硅表面, 即可制备 Janus 微球. 增加 对裸露的二氧化硅表面刻蚀程度, 该部分球冠逐渐 变为非球形, 得到非球形, 如蘑菇状 Janus 颗粒(图 2(b)). 未被刻蚀的颗粒表面功能基团如双键得以保 留, 进行表面接枝聚合得到聚合物层, 控制聚合程度 调控有机相一侧聚合物含量, 进一步控制颗粒的 Janus 平衡性参数性质; 控制聚合动力学, 在有机相 一侧获得聚合物纳米花状等系列新结构的 Janus 颗粒 (图 2(c e)), 显著增强了 Janus 颗粒微结构和物质分区 的调控能力. 与 Granick 等以硅烷偶联剂改性裸露二 氧化硅外表面显著不同, 我们采用的界面化学刻蚀 方法除能调控微球形状, 还能保证微球保持单个不 聚集. 硅烷偶联剂过量时将发生自聚, 存在颗粒在界 面粘结的可能.

水-石蜡体系在降温过程中发生液-固转变, 在界 面处导致结构重组，限制了界面保护应用. 而液相的 选择却极其丰富, 原理上只要两个液相不相溶, 即可 对界面上的胶体颗粒进行分区保护. 如何解决胶体 颗粒液-液界面运动, 特别是转动, 是充分利用 Pickering 乳液界面制备 Janus 颗粒的关键问题 ${ }^{[50]}$. 一 旦在改性过程中胶体发生转动, 将会影响胶体表面 的精确分区改性，甚至导致 Janus 胶体制备的失败. 我们提出了一步制备 Janus 颗粒的方法, 即油-水两相 同时进行原子转移自由基聚合(ATRP)在微球两面分 别接枝亲油和亲水聚合物(图 3) ) $^{[51]}$. 二氧化硅颗粒表 面用硅烷偶联剂亲油改性在表面获得苄基氯官能团, 可制备 W/O 型 Pickering 乳液. 在油相和水相中分别 存在不混溶的吡啶配体, 在铜离子与苄基氯共同作 用下, 在二氧化硅表面的两侧分别接枝聚苯乙烯(PS) 和聚丙烯酰胺(PAM), 制备了 Janus 颗粒. 聚合反应 一旦发生, 界面颗粒就具备了 Janus 特性, 抑制了其 在界面的转动, 确保了颗粒表面组成的严格分区. 对 该 Janus 颗粒表面特定区域进一步复合功能物质, 如 在 PAM 区域通过特殊作用选择性吸附纳米粒子(如 
(a)

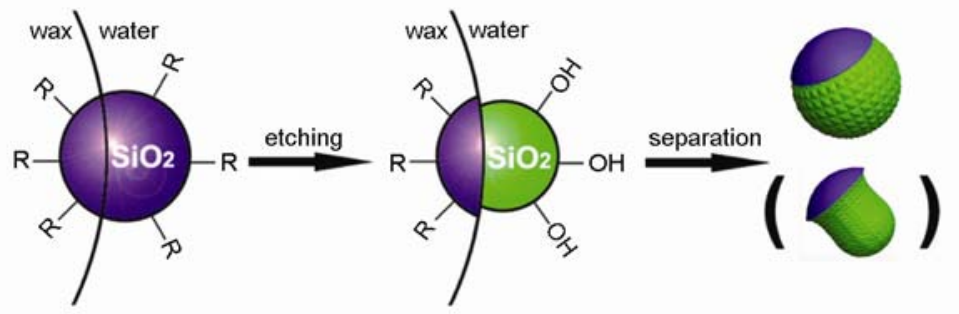

(b)

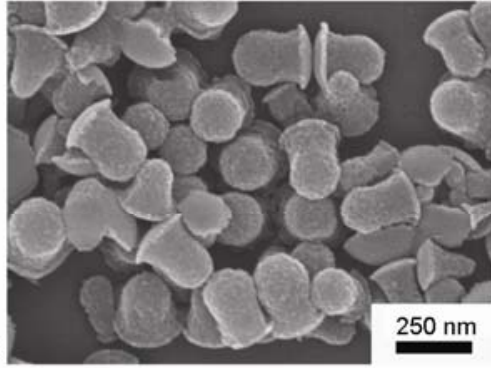

(d)

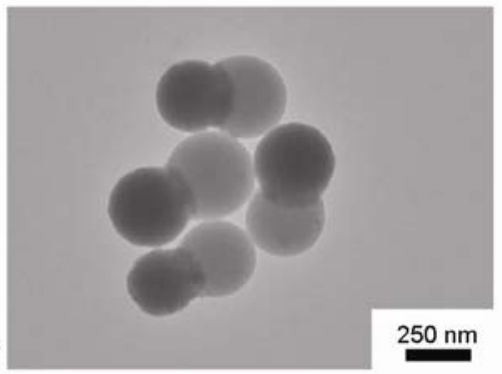

(c)

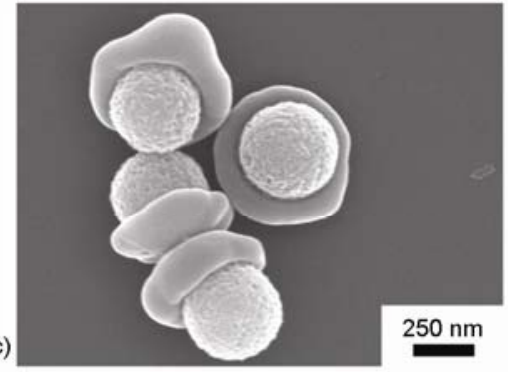

(e)

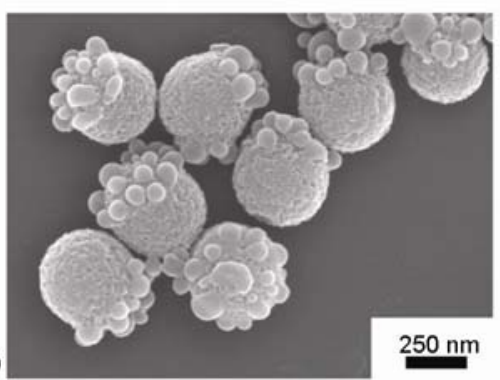

图 2 (a) 非球形 Janus 颗粒制备示意图. 不同形貌的非球形 Janus 颗粒: (b) 蘑菇状; (c) 带帽状; (d) 哑铃状; (e) 纳米花状 ${ }^{[49]}$

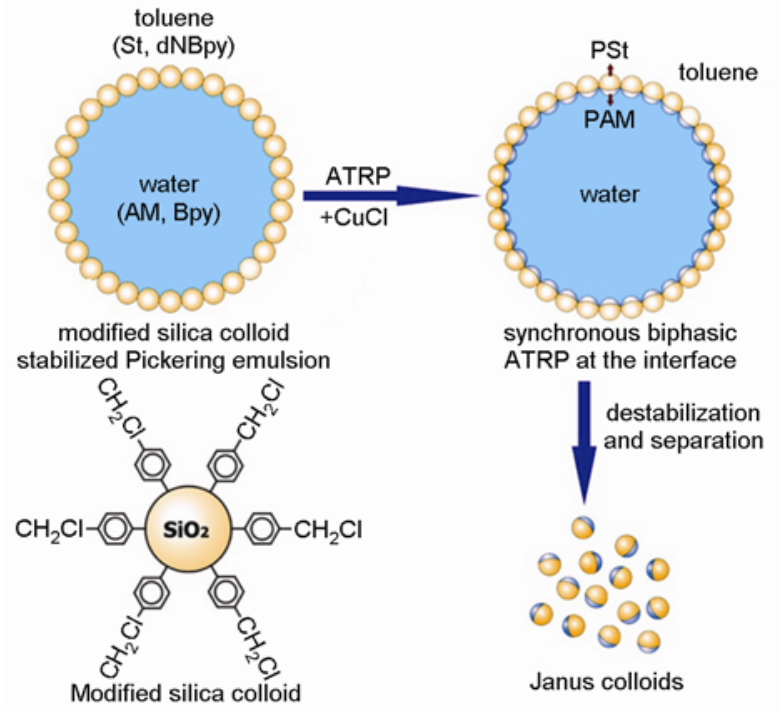

图 3 Pickering 乳液双相接枝制备 Janus 颗粒 ${ }^{[51]}$

金属 $\mathrm{Au}$ 、顺磁性 $\mathrm{Fe}_{3} \mathrm{O}_{4}$ ), 或吸附 $\mathrm{Ag}^{+}$并原位还原生成 $\mathrm{Ag}$ 纳米粒子等, 可衍生系列 Janus 复合功能颗粒. 除
了拓展了化学组成, 该区域的微结构也发生了相应 变化.

然而，上述工作中均使用已经制备的颗粒通过 化学改性使其稳定于 Pickering 乳液界面并实现分区, 制备出的 Janus 颗粒形变能力有限. 我们发展了联合 传统乳液和原位生成颗粒 Pickering 效应的制备方法, 得到组成严格分区和形状大幅度可控的 Janus 颗粒 ${ }^{[52]}$. 对于传统乳液, 在分散油相中进行自由基聚合导致 相分离获得可形变聚合物微粒, 由于 Pickering 效应 向界面扩散并稳定在界面处, 在界面的裸露程度可 控; 调节其在界面处三相线的界面张力差，控制聚合 物颗粒在界面拉伸形变程度，即可调节颗粒非球型 程度(飞碟状、半球、算盘珠等)(图 4). 在颗粒朝向水 相一侧进行亲水单体的聚合, 可获得 Janus 特性. 进 一步利用亲水聚合物与功能物质的特殊作用，复合 其他功能物质如 $\mathrm{Fe}_{3} \mathrm{O}_{4}, \mathrm{SiO}_{2}$, 则可实现组成和微结 构的精确调控. 


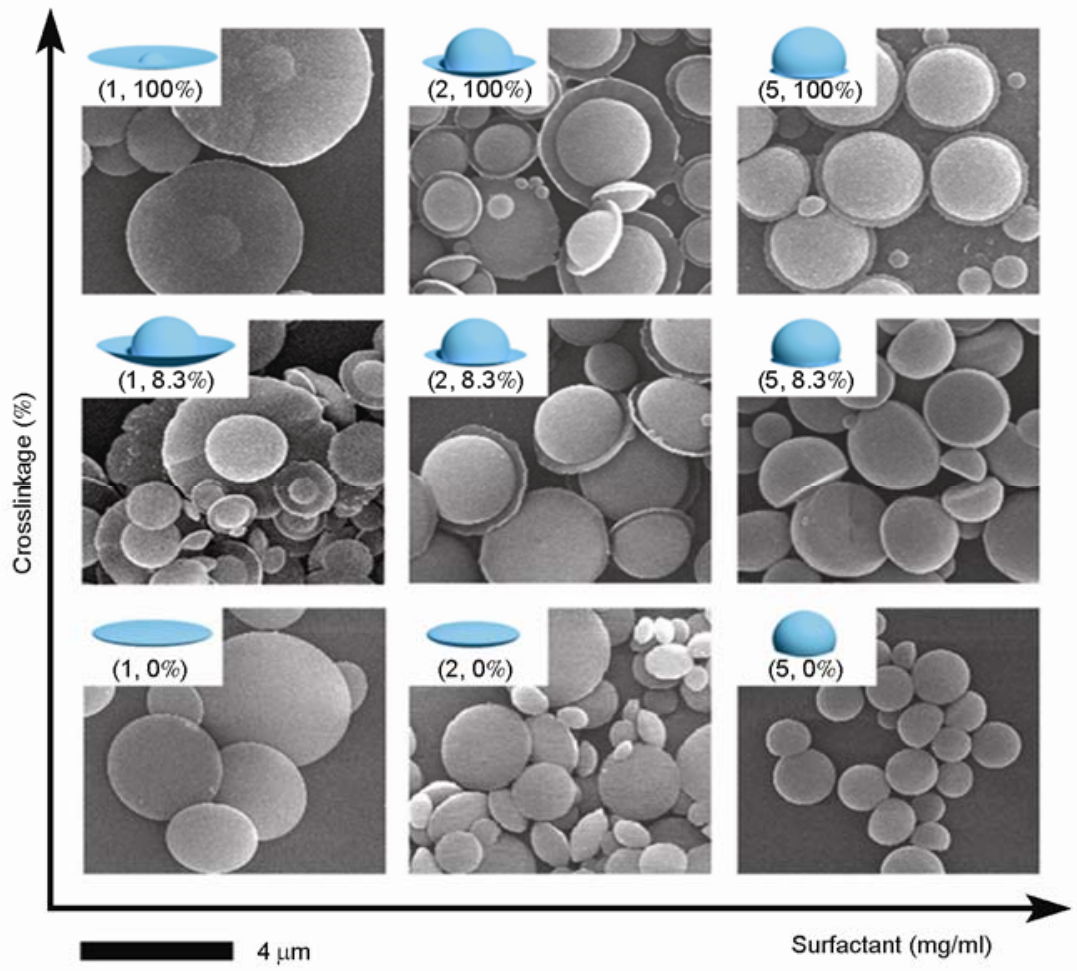

图 4 不同形貌的飞碟状 Janus 颗粒 ${ }^{[52]}$

\section{2 种子乳液聚合大规模制备 Janus 颗粒}

尽管上述方法能调控 Janus 颗粒的组成和微结构, 但仍然没有解决其批量制备的问题. 为此我们发展了 两种批量制备在组成和结构严格意义的 Janus 颗粒的 方法.

将表面润湿-去润湿原理应用于球面, 种子乳液 聚合制备 $\mathrm{SiO}_{2} / \mathrm{PS}$ 复合非球型 Janus 颗粒(图 5(a)), 聚 合诱导相分离和交联辅助相分离是控制非球型关键, 该方法具有普适性 ${ }^{[33,54]}$. 该类颗粒在 $\mathrm{SiO}_{2}$ 占优一侧 仍存在极薄的聚合物层. 尽管人们经常称该类颗粒 为 Janus, 但从化学组成方面, 该颗粒不是严格意义
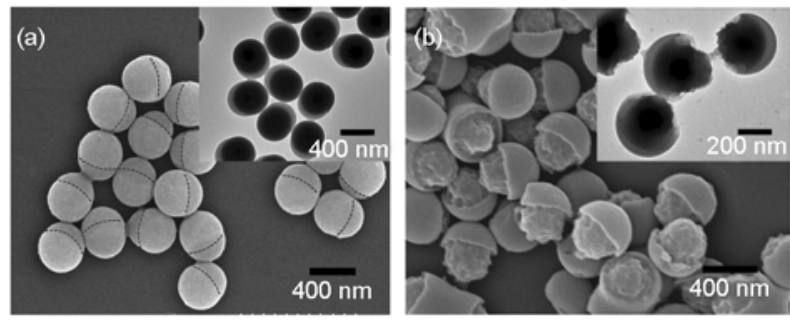

图 $5 \mathrm{SiO}_{2} / \mathrm{PS}$ 复合非球型颗粒刻蚀前(a)后(b)获得的结构和 组成严格意义的 Janus 颗粒 ${ }^{[55]}$
的 Janus 颗粒. 我们通过超声辅助表面刻蚀去除聚合 物薄层重新露出新鲜 $\mathrm{SiO}_{2}$ 表面(图 5(b)) 获得了组成严 格分区的 Janus 颗粒; 进一步以硅烷偶联剂选区改性 以及功能物质复合, 可拓展 Janus 颗粒组成 ${ }^{[55]}$. 控制 无机部分刻蚀程度, 即可实现 Janus 亲水亲油平衡性 调控.

除了无机颗粒，基于聚合诱导多组分聚合物相 分离原理，利用溶胀聚合技术，是制备非球型颗粒的 通用方法 ${ }^{[56,57]}$. 类似地, 传统方法制备的颗粒虽然在 形状上具有非对称性，也经常被认为是 Janus，但由 于相分离经常导致富相中还有少量另外组分, 所以 从化学组成角度, 该类非球型颗粒不是严格意义的 Janus 颗粒. 我们以制备非球型 PAN/PS 复合中空结 构为例, 选用交联结构 PAN 为高分子基体, 保证了 单体溶胀聚合导致的 PS 部分不含有 PAN; 通过 PAN 水解衍生官能团并利用高分子凝胶诱导功能物质选 区优先生长, 得到系列 Janus 复合颗粒(包括 $\mathrm{SiO}_{2} / \mathrm{PS}$ 、 $\mathrm{TiO}_{2} / \mathrm{PS} 、 \mathrm{Fe}_{3} \mathrm{O}_{4} / \mathrm{PS}$ 等)(图 6) ${ }^{[58]}$, 从形状和组成两方 面均具备了严格的 Janus 分区性质. 控制单体量, 调 节 Janus 颗粒两部分比例, 则可调控其 Janus 平衡值. 该方法具有批量制备的优点. 选用 $\mathrm{Fe}_{3} \mathrm{O}_{4} / \mathrm{PS}$ 磁性 

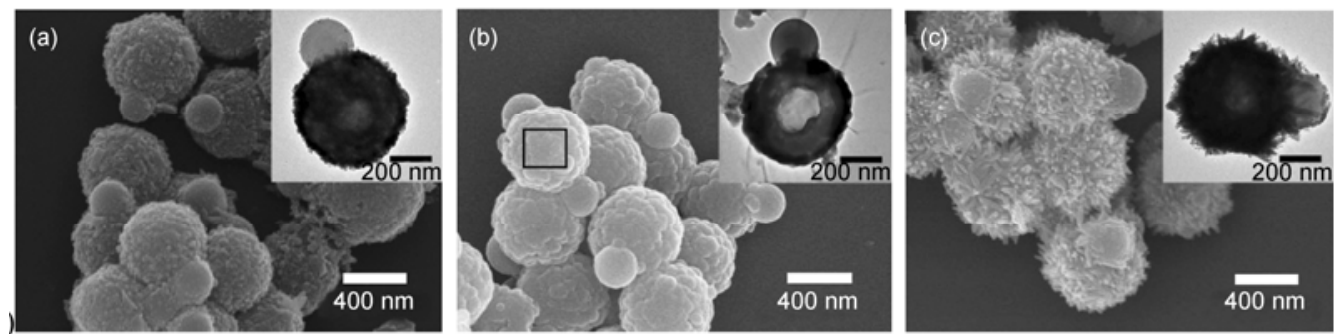

图 6 组成和微结构可调的 Janus 中空球. (a) $\mathrm{SiO}_{2} / \mathrm{PS}$; (b) $\mathrm{TiO}_{2} / \mathrm{PS}$; (c) $\mathrm{Fe}_{3} \mathrm{O}_{4} / \mathrm{PS}^{[59]}$

Janus 颗粒，可有效稳定油/水界面制备乳液，颗粒在 界面具有明确指向, 分散相液滴可在磁场作用下驱动.

\subsection{Janus 新型结构}

目前报道的 Janus 材料大多为球形, 我们的研究 则从球形颗粒拓展到其他复杂结构如 Janus 中空球和 Janus 片等形态.

利用乳液界面的双亲特性, 在界面处进行自组 织的溶胶-凝胶过程, 可实现界面的材料化, 制得外 表面亲水、内表面亲油的无机 Janus 中空球, 球壳厚 度在纳米尺度可调. 在去除油相时产生的渗透压导 致在球壳上形成孔通道, 保证了物质的输运(图 7 ${ }^{[59]}$. 与普通的壳层内外性质相同的中空球相比, Janus 中 空球在药物可控释放、受限反应和催化方面均将具有
优势.

该类结构类似稳定的胶束结构, 具备选择性地 从水相中富集油的特性, 从功能上可以称之为 Janus 容器(图 8(a)). 当油水乳液经过由此 Janus 中空球组 成的填料柱时, 乳液中的油相被吸入 Janus 球的亲油 空腔内, 而水相则排出, 实现了高效油水分离(图 8(b, c)).

研磨上述 Janus 中空球，得到了具有双亲性的 Janus 纳米片状材料(图 9(b)). 此 Janus 片作为固体乳 化剂可以很好地稳定油水乳液(图 9(c)). 与传统固体 乳化剂相比, Janus 片具有高度各向异性和更强的界 面动态稳定性质, 稳定的液滴在空气中高度稳定不 破乳 ${ }^{[60]}$.

由于分散相液滴尺度大，采用过滤的方法便可

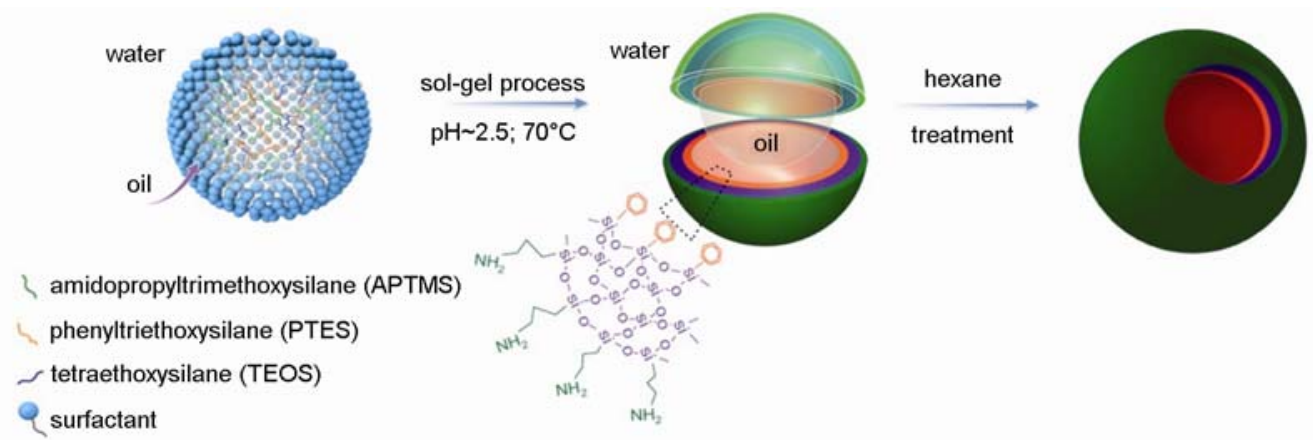

图 7 通过乳液界面自组装的溶胶-凝胶过程制备 Janus 中空球 ${ }^{[59]}$
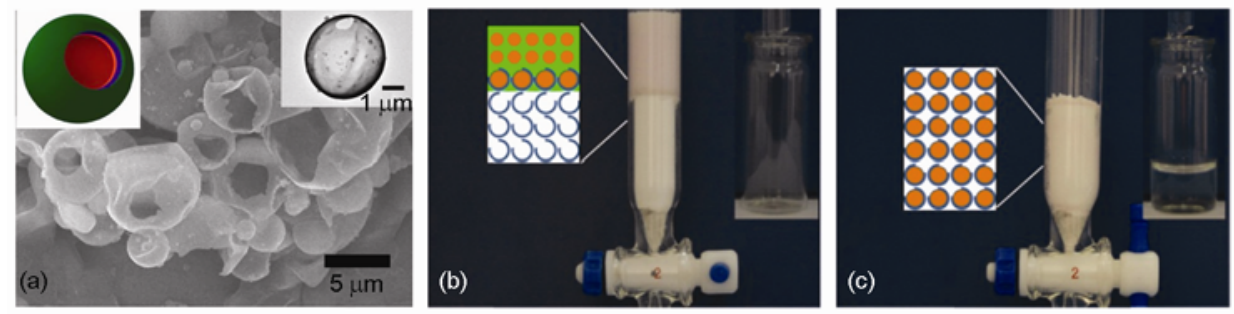

图 8 (a) 无机 Janus 中空球; (b, c) Janus 中空球填料柱用于油水分离 [59] 
(1)

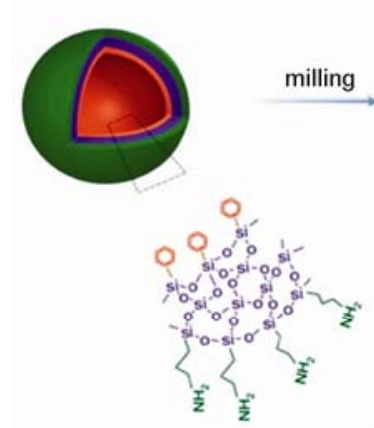

(2)

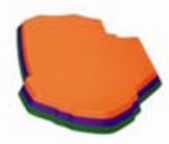

oil droplet

stabilized with Janus nanosheets
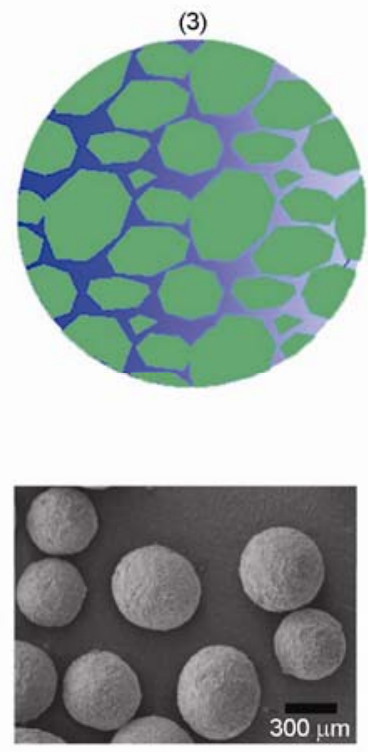

图 9 Janus 中空球通过破碎的方法得到 Janus 纳米片, 并用作颗粒乳化剂稳定油/水界面制备乳液体系 ${ }^{[60]}$
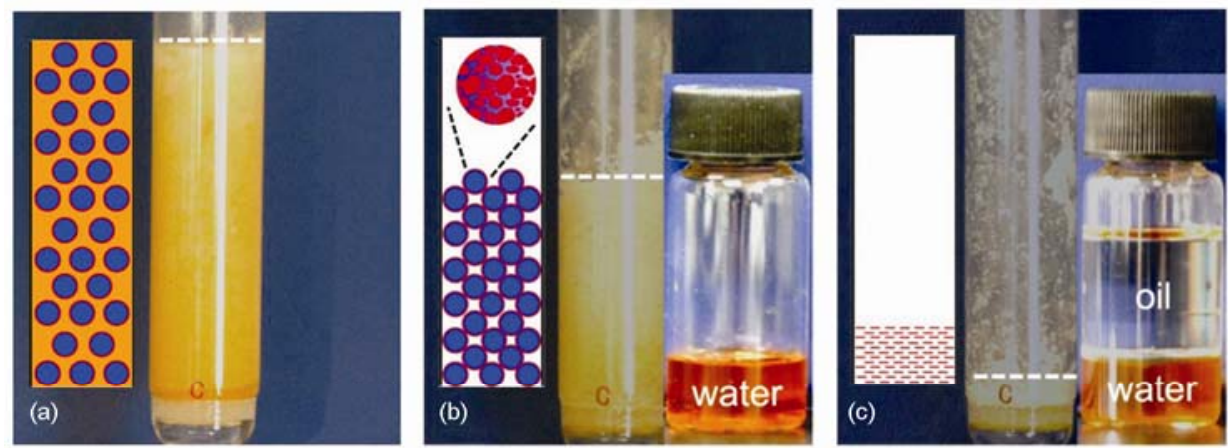

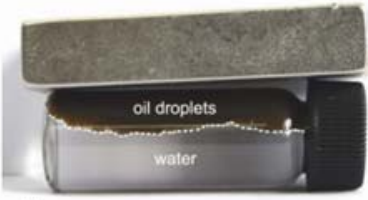

(d)

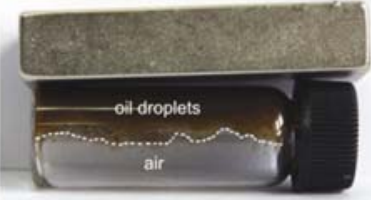

(e)

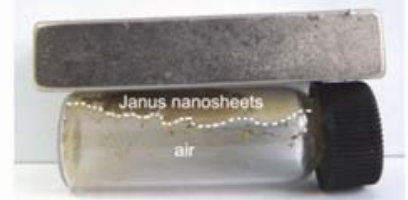

(f)

图 10 (a c) Janus 纳米片材料稳定 $\mathrm{O} / \mathrm{W}$ 型乳液并用于油水分离; $(\mathrm{d} \sim \mathrm{f})$ 磁性 Janus 复合纳米片用于乳液液滴的磁操纵和 Janus 复合片的磁回收 ${ }^{[60]}$

简单地实现乳液中油水两相分离(图 10(a c)). 将甲 苯为分散相、水为连续相的乳液加入到以多孔玻璃砂 材料为底的分离柱中, 连续相水经过玻璃砂流出, 而 甲苯在 Janus 纳米片的稳定下形成的大液滴不能通过 玻璃砂底部的孔流出, 仍保留在分离柱中. 用玻璃棒 将分离柱中 Janus 片稳定的油滴捣碎，甲苯经过玻璃 砂底流出, Janus 片保留在分离柱中, 实现了乳液的油
水两相的分离和 Janus 片的回收. 特别有趣的是, 甲 苯的大液滴在空气中稳定, 这归因于高度各向异性 的 Janus 纳米片在界面的高度动力学稳定行为. 通过 选择生长物质可赋予其功能性, 如在亲水一侧吸附 磁性 $\mathrm{Fe}_{3} \mathrm{O}_{4}$ 纳米粒子得到了磁响应性的 Janus 复合纳 米片. 不改变其润湿性, 进而赋予磁性 Janus 复合片 稳定的乳液磁响应性, 可实现对乳液的磁操纵和 
Janus 片的磁回收(图 10(d f)). 利用此特性, Janus 复 合纳米片材料将在在污水处理和有机物遗撒应急处 理方面具有应用前景.

环状材料是一种特殊的腔体材料, 具有开放性 的内部空腔以及封闭的环形通路. 我们结合超薄切 片技术，发展了一种制备亚微米尺度 Janus 环状材料 的普适方法(图 11) ${ }^{[61]}$. 以多孔氧化铝膜为模板, 利用 ATRP 聚合技术，在其孔通道中制得了聚苯乙烯纳米 管; 通过官能团改性、功能物质复合等过程, 拓展内

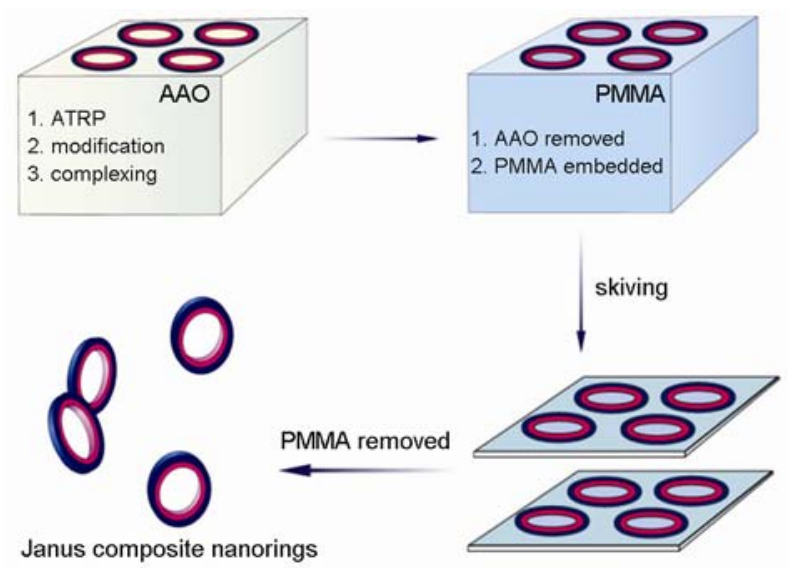

图 11 Janus 环制备过程示意图 ${ }^{[61]}$
外表面组成制备了不同的复合双层纳米管，如 $\mathrm{TiO}_{2} / \mathrm{PS}$, PANi/PS 纳米管等. 对上述纳米管进行超薄切片, 得 到相应组成的 Janus 环状材料. 控制切片步长, 可精 确控制环的高度. 该方法得到的 Janus 环状材料组成 和特征尺度可控.

\section{3 总结与展望}

具有 Janus 结构的材料具有优异的性能和有趣的 物理化学现象, 日益受到科学界的重视. Janus 颗粒的 制备研究进展迅速, 从形貌到组成都得到了极大的 拓展，为其在诸多领域的应用提供了坚实基础. 我们 围绕着 Janus 颗粒组成、形貌及化学组成严格分区和 微结构的精细调控新方法和其大批量制备等方面取 得了进展, 为 Janus 颗粒综合性质研究和应用提供了 新机会. 但是, 在 Janus 粒子合成的方法学方面仍面 临挑战, 如发展纳米尺度的 Janus 粒子大量合成和功 能化的普适性方法, Janus 棒和盘结构精细控制和大 量合成的普适性方法. 另外, Janus 粒子与双亲分子的 作用与共组装行为的深入研究, 将有助于揭示物质 作用和聚集行为的本质, 为新型功能材料的设计提 供新思路.

\section{参考文献}

1 Perro A, Reculusa S, Ravaine S, Bourgeat-Lamic E, Duguet E. Design and synthesis of Janus micro- and nanoparticles. J Mater Chem, 2005, 15(35-36): 3745-3760

2 Yang SM, Kim SH, Lim JM, Yi GR. Synthesis and assembly of structured colloidal particles. J Mater Chem, 2008, 18(9): 2177-2190

3 Walther A, Müller AHE. Janus particles. Soft Matter, 2008, 4(4): 663-668

4 Wang C, Xu CJ, Zeng H, Sun SH. Recent progress in syntheses and applications of dumbbell-like nanoparticles. Adv Mater, 2009, 21(30): 3045-3052

5 Jiang S, Chen Q, Tripathy M, Luijten E, Schweizer KS, Granick S. Janus particle synthesis and assembly. Adv Mater, 2010, 22(10): 1060-1071

6 Lattuada M, Hatton TA. Synthesis, properties and applications of Janus nanoparticles. Nano Today, 2011, 6(3): 286-308

7 Pickering SU. Emulsions. J Chem Soc, 1907, 91: 2001-2021

8 Vignati E, Piazza R, Lockhart TP. Pickering emulsions: Interfacial tension, colloidal layer morphology, and trapped-particle motion. Langmuir, 2003, 19(17): 6650-6656

9 Binks BP, Lumsdon SO. Pickering emulsions stabilized by monodisperse latex particles: Effects of particle size. Langmuir, 2001, 17(15): $4540-4547$

10 Binks BP, Fletcher PDI. Particles adsorbed at the oil-water interface: A theroretical comparison between spheres of uniform wettability and "Janus" particles. Langmuir, 2001, 17(15): 4708-4710 
11 Takahara YK, Ikeda S, Ishino S, Tachi K, Ikeue K, Sakata T, Hasegawa T, Mori H, Matsumura M, Ohtani B. Asymmetrically modified silica particles: A aimple particulate surfactant for stabilization of oil droplets in water. J Am Chem Soc, 2005, 127(17): 6271-6275

12 Glaser N, Adams DJ, Boker A, Krausch G. Janus particles at liquid-liquid interfaces. Langmuir, 2006, 22(12): 5227-5229

13 Walther A, Hoffmann M, Müller AHE. Emulsion polymerization using Janus particles as stabilizers. Angew Chem Int Ed, 2008, 47(4): 711-714

14 Walther A, Matussek K, Müller AHE. Engineering nanostructured polymer blends with controlled nanoparticle location using Janus particles. ACS Nano, 2008, 2(6): 1167-1178

15 Nonomura Y, Komura S, Tsujii K. Adsorption of disk-shaped Janus beads at liquid-liquid interfaces. Langmuir, 2004, 20(26): 11821-11823

16 Nonomura Y, Komura S, Tsujii K. Adsorption of microstructured particles at liquid-liquid interfaces. J Phys Chem B, 2006, 110(26): $13124-13131$

17 Jiang S, Granick S. Janus balance of amphiphilic colloidal particles. J Chem Phys, 2007, 127(16): 161102-161104

18 Jiang S, Granick S. Controlling the geometry (Janus balance) of amphiphilic colloidal particles. Langmuir, 2008, 24(6): 2438-2445

19 Golestanian R, Liverpool TB, Ajdari A. Repulsion of a molecular machine by asymmetric distribution of reaction products. Phys Rev Lett, 2005, 94(22): 220801-220804

20 Howse JR, Jones RAL, Ryan AJ. Self-motile colloidal particles: from directed propulsion to random walk. Phys Rev Lett, 2007, 99(4): 048102-048105

21 Kagan D, Calvo-Marzal P, Balasubramanian S, Sattayasamitsathit S, Manesh KM, Flechsig GU, Wang J. Chemical sensing based on catalytic nanomotors: Motion-based detection of trace silver. J Am Chem Soc, 2009, 131(34): 12082-12083

22 Burdick J, Laocharoensuk R, Wheat PM, Posner JD, Wang J. Synthetic nanomotors in microchannel networks: Directional microchip motion and controlled manipulation of cargo. J Am Chem Soc, 2008, 130(26): 8164-8165

23 Sundararajan S, Lammert PE, Zudans AW, Crespi VH, Sen A. Catalytic motors for transport of colloidal cargo. Nano Lett, 2008, 8(5): $1271-1276$

24 Gibbs JG, Zhao YP. Autonomously motile catalytic nanomotors by bubble propulsion. Appl Phys Lett, 2009, 94(16): 163104-163106

25 Mirkovic T, Zacharia NS, Scholes GD, Ozin GA. Fuel for thought: chemically powered nanomotors out-swim nature's flagellated bacteria. ACS Nano, 2010, 4(4): 1782-1789

26 Himmelhaus M, Takei H. Cap-shaped gold nanoparticles for an optical biosensor. Sens Actuators B, 2000, 63(1-2): 24-30

27 Behrend J, Anker JN, McNaughton BH, Brasuel M, Philbert MA, Kopelman R. Metal-capped brownian and magnetically modulated optical nanoprobes (MOONs): Micromechanics in chemical and biological microenvironments. J Phys Chem B, 2004, 108(29): 10408-10414

28 Behrend CJ, Anker JN, Kopelman R. Brownian modulated optical nanoprobes. App Phys Lett, 2004, 84(1): 154-156

29 Anker JN, Behrend CJ, Kopelman EJ. Aspherical magnetically modulated optical nanoprobes (MagMOONs). Appl Phys, 2003, 93(10): 6698-6700

30 Choi J, Zhao Y, Zhang D, Chien S, Lo YH. Patterned fluorescent particles as nanoprobes for the investigation of molecular interactions. Nano Lett, 2003, 3(8): 995-1000

31 Xu C, Xie J, Ho D, Wang C, Kohler N, Walsh EG, Morgan JR, Chin YE, Sun S. Au-Fe $\mathrm{O}_{4}$ dumbbell nanoparticles as dual-functional probes. Angew Chem Int Ed, 2008, 47(1): 173-176

32 Nisisako T, Torii T, Takahashi T, Takizawa Y. Synthesis of monodisperse bicolored Janus particles with electrical anisotropy using a microfluidic co-flow system. Adv Mater, 2006, 18(9): 1152-1156

33 Hays DA. Paper documents via the electrostatic control of particles. J Electrostatics, 2001, 51: 57-63

34 Millman BK, Prevo BG, Velev OD. Anisotropic particle synthesis in dielectrophoretically controlled microdroplet reactors. Nat Mater, 2005, 4(1): 98-102

35 Fialkowski M, Bitner A, Grzybowski BA. Self-assembly of polymeric microspheres of complex internal structures. Nat Mater, 2005, 4(1): 93-97

36 Yin SN, Wang CF, Yu ZY, Wang J, Liu SS, Chen S. Versatile bifunctional magnetic-fluorescent responsive Janus supraballs towards the flexible bead display. Adv Mater, 2011, 23(26): 2915-2919

37 Chen Q, Whitmer JK, Jiang S, Bae SC, Luijten E, Granick S. Supracolloidal reaction kinetics of Janus spheres. Science, 2011, 331(6014): 199-202

38 Hong L, Cacciuto A, Luijten E, Granick S. Clusters of charged Janus spheres. Nano Lett, 2006. 6(11): 2510-2514

39 Hong L, Cacciuto A, Luijten E, Granick S. Clusters of amphiphilic colloidal spheres. Langmuir, 2008, 24(3): 621-625

40 Chen, Q, Bae SC, Granick S. Directed self-assembly of a colloidal kagome lattice. Nature, 2011, 469(7330): 381-384 
41 Chen Q, Diesel E, Whitmer JK, Bae SC, Luijten E, Granick S. Triblock colloids for directed self-assembly. J Am Chem Soc, 2011, 133(20): 7725-7727

42 Cho I, Lee KW. Morphology of latex-particles formed by poly(methyl methacrylate)-seeded emulsion polymerization of styrene. $J$ Appl Polym Sci, 1985, 30(5): 1903-1926

43 Casagrande C, Veyssie M. Janus beads-Realization and 1st observation of interfacial properties. Comptes Rendus L Acad Sci Ser I, 1988, 306(20): 1423-1425

44 Casagrande C, Raphaël PFE, Veyssié M. Janus beads-Realization and behavior at water oil interfaces. Europhys Lett, 1989, 9(3): 251-255

45 Hong L, Jiang S, Granick S. Simple method to produce Janus colloidal particles in large quantity. Langmuir, 2006, 22(23): $9495-9499$

46 Zerrouki D, Baudry J, Pine D, Chaikin P, Bibette J. Chiral colloidal clusters. Nature, 2008, 455: 380-382

47 Glotzer SC, Solomon MJ. Anisotropy of building blocks and their assembly into complex structures. Nat Mater, 2007, 6(8): 557-562

48 Gangwal S, Cayre OJ, Velev OD. Dielectrophoretic assembly of metallodielectric Janus particles in ac electric fields. Langmuir, 2008, 24(23): 13312-13320

49 Liu B, Zhang CL, Liu JG, Qu XZ, Yang ZZ. Janus non-spherical colloids by asymmetric wet-etching. Chem Commun, 2009: 3871-3873

50 Suzuki D, Tsuji S, Kawaguchi H. Janus microgels prepared by surfactant-free Pickering emulsion-based modification and their self-assembly. J Am Chem Soc, 2007, 129(26): 8088-8089

51 Liu B, Wei W, Qu XZ, Yang ZZ. Janus colloids formed by biphasic grafting at a Pickering emulsion interface. Angew Chem Int Ed, 2008, 47(21): 3973-3975

52 Wang YH, Zhang CL, Tang C, Li J, Shen K, Liu JG, Qu XZ, Li JL, Wang Q, Yang ZZ. Emulsion interfacial synthesis of asymmetric Janus particles. Macromolecules, 2011, 44(10): 3787-3794

53 Perro A, Reculusa S, Pereira F, Delville MH, Mingotaud C, Duguet E, Bourgeat-Lami E, Ravaine S. Towards large amounts of Janus nanoparticles through a protection-deprotection route. Chem Commun, 2005, 5542-5543

$54 \mathrm{Lu} \mathrm{W}$, Chen M, Wu LM. One-step synthesis of organic-inorganic hybrid asymmetric dimer particles via miniemulsion polymerization and functionalization with silver. J Colloid Interf Sci., 2008, 328(1): 98-102

55 Zhang CL, Liu B, Tang C, Liu JG, Qu XZ, Li JL, Yang ZZ. Large scale synthesis of Janus submicron sized colloids by wet etching anisotropic ones. Chem Commun, 2010, 46(25): 4610-4612

56 Kim JW, Larsen RJ, Weitz DA. Synthesis of nonspherical colloidal particles with anisotropic properties. J Am Chem Soc, 2006, 128(44): 14374-14377

57 Kim JW, Lee DY, Shum HC, Weitz DA. Colloid surfactants for emulsion stabilization. Adv Mater, 2008, 20(17): 3239-3243

58 Tang C, Zhang CL, Liu JG, Qu XZ, Li JL, Yang ZZ. Large scale synthesis of Janus submicrometer sized colloids by seeded emulsion polymerization. Macromolecules, 2010, 43(11): 5114-5120

59 Liang FX, Liu JG, Zhang CL, Qu XZ, Li Jiaoli, Yang ZZ. Janus hollow spheres by emulsion interfacial self-assembled sol-gel process. Chem Commun, 2011, 47(4): 1231-1233

60 Liang FX, Shen K, Qu XZ, Zhang CL, Wang Q, Li JL, Liu JG, Yang ZZ. Inorganic Janus nanosheets. Angew Chem Int Ed, 2011, 50(10): 2379-2382

61 Zhou Q, Li J, Zhang CL, Qu XZ, Liu JG, Li JL, Yang ZZ. Janus composite nanorings by combinational template synthesis and skiving micro-process. Polymer, 2010, 51(16): 3606-3611 


\title{
Progress on precise control of microstructure of Janus materials
}

\author{
ZHANG ChengLiang, WEI Wei, LIANG FuXin, YANG ZhenZhong*
}

State Key Laboratory of Polymer Physics and Chemistry; Institute of Chemistry, Chinese Academy of Sciences, Beijing 100190, China

*Corresponding author (email: yangzz@iccas.ac.cn)

\begin{abstract}
Janus materials with two different chemical regions compartmentalized onto the same objects are gaining growing interests. It has become a new hotspot of material research for its anisotropic composition and structure. It is key to develop new methods that can easily and large-scale synthesize Janus materials with tunable morphology, strict chemical composition partition, functional composite partition, and precise control of microstructure. It will support new ideas and measures for the further production and application of Janus materials
\end{abstract}

Keywords: Janus materials, anisotropic, precise control microstructure, large-scale synthesis 